\title{
REARRANGEMENTS OF SERIES OF FUNCTIONS
}

\author{
BY \\ VLADIMIR DROBOT
}

1. Introduction and the statement of the main result. It is well known [3, p. 301] that a conditionally convergent series of real numbers can be rearranged in such a way so as to converge to any preassigned value. Suppose now we have a series of functions

$$
\sum_{n=1}^{\infty} x_{n}(t), \quad 0 \leqq t \leqq 1
$$

In this paper we shall be concerned with the studies of rearrangements of such series, the convergence being that of $L_{2}(0,1)$. The work is motivated by the paper of E. Steinitz [4], who considered the rearrangements of conditionally convergent series of vectors in the finite dimensional Euclidean spaces. We are going to prove the following result:

THEOREM 1. Suppose $x_{n}(t)$ is a sequence of real valued functions, belonging to the real space $L_{2}(0,1)$. Suppose also that:

(a) the series (1) converges in norm to some $x \in L_{2}$,

(b) $\sum\left\|x_{n}\right\|=+\infty$,

(c) $\sum\left\|x_{n}\right\|^{2}<\infty$,

(d) the linear subspace $M=\left\{y \in L_{2}: \sum\left|\left(x_{n}, y\right)\right|<\infty\right\}$ is closed $((a, b)$ is the real inner product of $a$ and $b$ ).

Then there exists a closed linear subspace $N$ and a function $x_{0} \in L_{2}$ such that:

I. any rearrangement of (1), which converges in norm, must have the limit of the form $x_{0}+z$, where $z \in N$;

II. for any $z \in N$, there exists a rearrangement of (1), which converges in norm to $x_{0}+z$.

In fact $N=M^{\perp}$ i.e., $N \oplus M=L_{2}$.

2. Proof of Theorem.1. First we need several lemmas.

Lemma 1. Let $x_{1}, \ldots, x_{n+1}$ be $n+1$ linearly dependent elements in $L_{2}$ and let

$$
x=\alpha_{1} x_{1}+\cdots+\alpha_{n+1} x_{n+1}, \quad 0 \leqq \alpha_{i} \leqq 1 .
$$

Then we can express $x$ as

$$
x=\gamma_{1} x_{1}+\cdots+\gamma_{n+1} x_{n+1}, \quad 0 \leqq \gamma_{i} \leqq 1
$$

and at least one $\gamma_{i}=0$ or 1 .

Received by the editors November 20, 1968. 
Proof. This Lemma is proved in [4, p. 167] for the case when $x_{i} \in R^{n}$ and the proof carries verbatim to the present situation.

LEMMA 2. Let $x_{1}, x_{2}, \ldots, x_{n} \in L_{2}$ and let

$$
x=\lambda_{1} x_{1}+\cdots+\lambda_{n} x_{n}, \quad 0 \leqq \lambda_{1} \leqq 1 .
$$

Then there exists a vector $x^{\prime} \in L_{2}$ of the form

$$
x^{\prime}=\delta_{1} x_{1}+\delta_{2} x_{2}+\cdots+\delta_{n} x_{n}, \quad \delta_{i}=0 \text { or } 1
$$

such that $\left\|x-x^{\prime}\right\|^{2} \leqq\left\|x_{1}\right\|^{2}+\cdots+\left\|x_{n}\right\|^{2}$.

Proof. We proceed by induction on $n$. The case $n=1$ is clear since $\left|\lambda_{1}\right| \leqq 1$. Suppose then the lemma is true for any $n$ vectors in $L_{2}$ and let

$$
x=\lambda_{1} x_{1}+\cdots+\lambda_{n} x_{n}+\lambda_{n+1} x_{n+1}, \quad 0 \leqq \lambda_{i} \leqq 1 .
$$

We may write $x_{n+1}=y_{n+1}+z_{n+1}$, where $y_{n+1} \in \operatorname{sp}\left\{x_{1}, \ldots, x_{n}\right\}$ and $\left(z_{n+1}, x_{i}\right)=0$, $i=1,2, \ldots, n$. Clearly

$$
\begin{aligned}
\left\|x_{n+1}\right\|^{2} & =\left\|y_{n+1}\right\|^{2}+\left\|z_{n+1}\right\|^{2}, \\
x-\lambda_{n+1} z_{n+1} & =\lambda_{1} x_{1}+\cdots+\lambda_{n} x_{n}+\lambda_{n+1} y_{n+1}, \quad 0 \leqq \lambda_{i} \leqq 1
\end{aligned}
$$

and the vectors $x_{1}, \ldots, x_{n}, y_{n+1}$ are linearly dependent. Using Lemma 1 we can write (4) as

$$
x-\lambda_{n+\mathrm{i}} z_{n+1}=\gamma_{1} x_{1}+\cdots+\gamma_{n} x_{n}+\gamma_{n+1} y_{n+1}, \quad 0 \leqq \gamma_{i} \leqq 1
$$

and at least one $\gamma_{i_{0}}=0$ or 1 .

We divide the remaining proof into several cases.

Case 1. $i_{0}=n+1, \gamma_{n+1}=0$. By the inductive hypothesis there exists a vector $x^{\prime}=\delta_{1} x_{1}+\cdots+\delta_{n} x_{n}, \delta_{i}=0$ or 1 , such that

$$
\left\|x-\lambda_{n+1} z_{n+1}-x^{\prime}\right\|^{2} \leqq\left\|x_{1}\right\|^{2}+\cdots+\left\|x_{n}\right\|^{2} \text {. }
$$

Since $x=\left(x-\lambda_{n+1} z_{n+1}\right)+\lambda_{n+1} z_{n+1}$ and $z_{n+1}$ is orthogonal to $x-\lambda_{n+1} z_{n+1}$ (equation 5 and the conditions of this case) and $x^{\prime}$, we get from (6) and (3)

$$
\left\|x-x^{\prime}\right\|^{2}=\left\|x-\lambda_{n+1} z_{n+1}-x^{\prime}\right\|^{2}+\left|\lambda_{n+1}\right|^{2}\left\|z_{n+1}\right\|^{2} \leqq\left\|x_{1}\right\|^{2}+\cdots+\left\|x_{n+1}\right\|^{2} .
$$

Case 2. $i_{0}<n+1$ and $\gamma_{i_{0}}=0$. We may assume without the loss of generality that $i_{0}=1$. By the inductive hypothesis there is a vector $x^{\prime \prime}=\delta_{2} x_{2}+\cdots+\delta_{n+1} y_{n+1}$, $\delta_{i}=0$ or 1 , such that

$$
\left\|x-\lambda_{n+1} z_{n+1}-x^{\prime \prime}\right\| \leqq\left\|x_{2}\right\|^{2}+\cdots+\left\|x_{n}\right\|^{2}+\left\|y_{n+1}\right\|^{2} .
$$

Let $x^{\prime}=x^{\prime \prime}+\delta_{n+1} z_{n+1}=\delta_{2} x_{2}+\cdots+\delta_{n+1} x_{n+1}$. Since $z_{n+1}$ is orthogonal to $x_{1}, x_{2}$, $\ldots, x_{n}, y_{n+1}$, and $\left|\lambda_{n+1}-\delta_{n+1}\right| \leqq 1$ we obtain from (7) and (3)

$$
\begin{aligned}
\left\|x-x^{\prime}\right\|^{2} & =\left\|x-\lambda_{n+1} z_{n+1}-x^{\prime \prime}+\left(\lambda_{n+1}-\delta_{n+1}\right) z_{n+1}\right\|^{2} \\
& =\left\|x-\lambda_{n+1} z_{n+1}-x^{\prime \prime}\right\|^{2}+\left|\lambda_{n+1}-\delta_{n+1}\right|^{2}\left\|z_{n}\right\|^{2} \\
& \leqq\left\|x_{1}\right\|^{2}+\left\|x_{2}\right\|^{2}+\cdots+\left\|x_{n}\right\|^{2}+\left\|y_{n+1}\right\|^{2}+\left\|z_{n+1}\right\|^{2} .
\end{aligned}
$$

Since the last 2 terms add up to $\left\|x_{n+1}\right\|^{2}$ we get the result. 
Case 3. $i_{0}=n+1, \gamma_{n+1}=1$. Here

$$
x-\lambda_{n+1} z_{n+1}=\gamma_{1} x_{1}+\cdots+\gamma_{n+1} x_{n}+y_{n+1} .
$$

Let $x^{\prime \prime}=\delta_{1} x_{1}+\cdots+\delta_{n} x_{n}, \delta_{i}=0$ or 1 , be such that

$$
\left\|x-\lambda_{n+1} z_{n+1}-y_{n}-x^{\prime \prime}\right\|^{2} \leqq\left\|x_{1}\right\|^{2}+\cdots+\left\|x_{n}\right\|^{2}
$$

and put $x^{\prime}=x^{\prime \prime}+x_{n+1}=x_{n+1}+y_{n+1}+z_{n+1}$. Since $\left|\lambda_{n+1}-1\right| \leqq 1$ and $z_{i} \perp x_{i}, \ldots$, $x_{n}, y_{n+1}$, we get from (9) and (8)

$$
\begin{aligned}
\left\|x-x^{\prime}\right\|^{2} & =\left\|x-\lambda_{n+1} z_{n+1}-y_{n+1}-x^{\prime \prime}+\left(\lambda_{n+1}-1\right) z_{n+1}\right\|^{2} \\
& =\left\|x-\lambda_{n+1} z_{n+1}-y_{n+1}-x^{\prime \prime}\right\|^{2}+\left|\lambda_{n+1}-1\right|^{2}\left\|z_{n+1}\right\|^{2} \\
& \leqq\left\|x_{1}\right\|^{2}+\cdots+\left\|x_{n+1}\right\|^{2} .
\end{aligned}
$$

Case 4. $i<n+1$. Here again we assume that $i_{0}=1$ and consequently $\gamma_{1}=1$. We write now

$$
x-\lambda_{n+1} z_{n+1}-x_{1}=\gamma_{2} x_{2}+\cdots+\gamma_{n} x_{n}+\gamma_{n+1} y_{n+1} .
$$

Let $x^{\prime \prime}=\delta_{2} x_{2}+\cdots+\delta_{n} x_{n}+\delta_{n+1} y_{n+1}, \delta_{i}=0$ or 1 , be such that

$$
\left\|x-\lambda_{n+1} z_{n+1}-x_{1}-x^{\prime \prime}\right\| \leqq\left\|x_{2}\right\|^{2}+\cdots+\left\|y_{n+1}\right\|^{2} \text {. }
$$

Put

$$
x^{\prime}=x_{1}+x^{\prime \prime}+\delta_{n+1} z_{n+1}=x_{1}+\delta_{2} x_{2}+\cdots+\delta_{n+1} x_{n+1} .
$$

Noting again that $z_{n+1} \perp x_{1}, \ldots, y_{n+1},\left|\lambda_{n+1}-\delta_{n+1}\right| \leqq 1$ we get

$$
\begin{aligned}
\left\|x-x^{\prime}\right\|^{2} & =\left\|x-\lambda_{n+1} z_{n+1}-x_{1}-x^{\prime \prime}+\left(\lambda_{n+1}-\delta_{n+1}\right) z_{n+1}\right\|^{2} \\
& \leqq\left\|x_{2}\right\|^{2}+\cdots+\left\|y_{n+1}\right\|^{2}+\left|\lambda_{n+1}-\delta_{n+1}\right|^{2}\left\|z_{n+1}\right\|^{2} \leqq\left\|x_{1}\right\|^{2}+\cdots+\left\|x_{n+1}\right\|^{2} .
\end{aligned}
$$

This proves Lemma 2.

LEMMA 3. Let $X=\left\{x_{n}\right\}$ be a sequence of elements of $L_{2}$. Let

$$
\begin{aligned}
& P(X)=\left\{x_{i_{1}}+x_{i_{2}}+\cdots+x_{i_{k}}: i_{1}<i_{2}<\cdots<i_{k}\right\}, \\
& Q(X)=\operatorname{co} P(X) \quad(\text { convex hull of } P(X)) . \\
& R(X)=\left\{\gamma_{1} x_{i_{1}}+\cdots+\gamma_{k} x_{i_{k}}: 0 \leqq \gamma_{i} \leqq 1, i_{1}<i_{2}<\cdots<i_{k}\right\} .
\end{aligned}
$$

Then $Q(X) \subset R(X)$.

Proof. It is enough to show that $R(X)$ is convex, since $R(X) \supset P(X)$. Let now $y, z \in R(X)$. We may assume

$$
y=\gamma_{1} x_{i_{1}}+\gamma_{2} x_{i_{2}}+\cdots+\gamma_{k} x_{i_{k}}, \quad z=\delta_{1} x_{i_{1}}+\delta_{2} x_{i_{2}}+\cdots+\delta_{k} x_{i_{k}}
$$

by inserting the terms $0 \cdot x_{j}$ if necessary.

Let now $0 \leqq \lambda \leqq 1$. We have

$$
\lambda y+(1-\lambda z)=\sum_{j}\left[\lambda \gamma_{j}+(1-\lambda) \delta_{j}\right] x_{i_{j}}
$$

and since $0 \leqq \lambda \gamma_{j}+(1-\lambda) \delta_{j} \leqq \lambda+(1-\lambda)=1$ we get the result. 
LEMMA 4. Let $N$ be a closed linear subspace of $L_{2}$ and let $B$ be a convex subset of $N$. Suppose that for any $x \in N$ and any $T>0$, there exist elements $b_{1}$ and $b_{2}$ in $B$ so that $\left(x, b_{1}\right) \leqq-T$ and $\left(x, b_{2}\right) \geqq T$. Then $B$ is dense in $N$. (We recall that our $L_{2}$ is a real space.)

Proof. Consider $N$ as a Hilbert space and suppose the closure of $B$ is different from $N$. Let $K=\left\{x \in N:\left\|x-x_{0}\right\|<r\right\} \subset N \backslash \bar{B}$, for some $x_{0} \in N$ and $r>0$. Since $K$ and $\bar{B}$ are convex and $K$ has an interior point (in the relative topology of $N$ ), there exists a continuous linear functional $x^{\prime}$ such that $x^{\prime}(x) \leqq c \leqq x^{\prime}(y)$, for all $x \in \bar{B}, y \in K$ for some constant $c$. (See [2, p. 412].) By the Riesz representation theorem for the Hilbert spaces, $x^{\prime}(x)=\left(x, x^{\prime}\right)$ for some $x^{\prime} \in N$. But this implies that $\left(x, x^{\prime}\right) \leqq c$ for all $x \in B$, which contradicts the hypothesis of the theorem.

Lemma 5. Suppose $x_{i} \in L_{2},\left\|x_{i}\right\| \leqq M, i=1,2, \ldots, n$. Let

$$
x_{1}+x_{2}+\cdots+x_{n}=a \text {. }
$$

Then we can rearrange the order of $x_{i}^{\prime} s$, say into $\left\{x_{1}^{\prime}, x_{2}^{\prime}, \ldots, x_{n}^{\prime}\right\}$, such that

$$
\left\|x_{1}^{\prime}+x_{2}^{\prime}+\cdots+x_{p}^{\prime}\right\|^{2} \leqq\left\|x_{1}\right\|^{2}+\cdots+\left\|x_{n}\right\|^{2}+\|a\|(\|a\|+2 M), p=1,2, \ldots, n .
$$

Proof. First assume that $a=0$ and call the right hand side of (12) $K$. We proceed to rearrange $x_{1}, \ldots, x_{n}$ as follows. Let $x_{1}^{\prime}=x_{1}$. Clearly $\left\|x_{1}^{\prime}\right\|^{2} \leqq K$. On account of (11) we have $\sum\left(x_{1}^{\prime}, x_{1}\right)=\left(x_{1}^{\prime}, 0\right)=0$ and the first term of the sum is equal to $\left\|x_{1}^{\prime}\right\|^{2} \geqq 0$. Hence for some $x_{2}^{\prime}$ among $x_{2}, \ldots, x_{n}$ we must have $\left(x_{1}^{\prime}, x_{2}^{\prime}\right) \leqq 0$. From this it follows that

$$
\left\|x_{1}^{\prime}+x_{2}^{\prime}\right\|^{2}=\left\|x_{1}^{\prime}\right\|^{2}+2\left(x_{1}^{\prime}, x_{2}^{\prime}\right)+\left\|x_{2}^{\prime}\right\|^{2} \leqq\left\|x_{1}^{\prime}\right\|^{2}+\left\|x_{2}^{\prime}\right\|^{2} \leqq K .
$$

Consider next $\sum\left(x_{1}^{\prime}+x_{2}^{\prime}, x_{i}\right)=\left(x_{1}^{\prime}+x_{2}^{\prime}, 0\right)=0$. The first 2 terms add up to $\left\|x_{1}^{\prime}+x_{2}^{\prime}\right\|^{2}$ $\geqq 0$; hence for some $x_{3}^{\prime}$ among $x$ 's different from $x_{1}^{\prime}, x_{2}^{\prime}$ we must have $\left(x_{1}^{\prime}+x_{2}^{\prime}, x_{3}^{\prime}\right)$ $\leqq 0$. So

$$
\left\|x_{1}^{\prime}+x_{2}^{\prime}+x_{3}^{\prime}\right\|^{2}=\left\|x_{1}^{\prime}+x_{2}^{\prime}\right\|^{2}+2\left(x_{1}^{\prime}+x_{2}^{\prime}, x_{3}^{\prime}\right)+\left\|x_{3}^{\prime}\right\|^{2} \leqq K .
$$

Continuing in this fashion we get the result.

Suppose now $a \neq 0$. Then $\sum\left(x_{i}-n^{-1} a\right)=0$; so we can order $x_{i}$ 's in such a way that

$$
\begin{aligned}
\left\|\sum_{1}^{p}\left(x_{1}^{\prime}-\frac{1}{n} a\right)\right\|^{2} & \leqq \sum_{1}^{n}\left\|x_{i}-\frac{1}{n} a\right\|^{2} \leqq \sum_{1}^{n}\left(\left\|x_{i}\right\|^{2}+2 \frac{1}{n}\left\|x_{i}\right\|\|a\|+\frac{1}{n^{2}}\|a\|^{2}\right) \\
& \leqq(2 M+\|a\|)\|a\|+\sum\left\|x_{i}\right\|^{2} .
\end{aligned}
$$

We are now ready to prove Theorem 1 . Let $X=\left\{x_{n}: n=1,2, \ldots\right\}$ be the sequence of functions satisfying the conditions a-d. Each $x_{n}$ can be written as $x_{n}=y_{n}+z_{n}$, $y_{n} \in M, z_{n} \in M^{\perp}=N$. It is clear that if $\left\{x_{n}^{\prime}\right\}$ is a rearrangement of $X$, then $\sum x_{n}^{\prime}$ 
converges in $L_{2}$ if and only if $\sum y_{n}^{\prime}$ and $\sum z_{n}^{\prime}$ converge in $L_{2}$. We shall show first that $\sum y_{n}^{\prime}$ converges for any rearrangements of $y$ 's. For any $y \in M$ we have

$$
\sum\left|\left(y_{n}, y\right)\right|=\sum\left|\left(x_{n}, y\right)\right|<\infty \text {. }
$$

Hence $\sum\left(y_{n}, y\right)$ converges absolutely, so every rearrangement of $\sum\left(y_{n}, y\right)$ will converge to the same limit, say $W(y)$. This shows that $\sum y_{n}$ converges weakly, and for every $y \in M$, every subseries of $\sum\left(y_{n}, y\right)$ will also converge. This implies $\left[1\right.$, p. 60] that every subseries of $\sum y_{n}$ will converge, which in turn implies $[1$, p. 59 (1-b)] that every rearrangement of $\sum y_{n}$ converges in norm. The strong limit of $\sum y_{n}$ must be equal to the weak limit of $\sum y_{n}$. The weak limit of $\sum y_{n}$ is the same for every rearrangement, hence the strong limit must be independent of the rearrangement. This shows that $\sum y_{n}$ converges unconditionally in norm, i.e. for every rearrangement it converges in norm to the same limit, say $x_{0}$. This proves the first part of the theorem, since if a rearrangement of $\sum x_{n}$ converges in norm then the limit must be of the form $x_{0}+\sum z_{n}^{\prime}$ where $\sum z_{n}^{\prime} \in N=M^{\perp}$.

What remains to be shown is that for every $w \in N$ there exists a rearrangement of $\sum z_{n}$ which converges in norm to $w$. We introduce the following notation.

Let $W=\left\{w_{i}: i=1,2, \ldots\right\}$ be an arbitrary sequence of elements in $N$. Put

$P(W)=\left\{w_{i_{1}}+w_{i_{2}}+\cdots+w_{i_{k}}: i_{1}<i_{2}<\cdots<i_{k}\right\}$,

$Q(W)=\operatorname{co} P(W) \quad$ (convex hull of $P(W)$ ),

$R(W)=\left\{\gamma_{1} w_{i_{1}}+\cdots+\gamma_{k} w_{\imath_{k}}: 0 \leqq \gamma_{i} \leqq 1, i_{1}<i_{2}<\cdots<i_{k}\right\}$.

Denote the elements of $P(W)$ by $p$. If $p=w_{i_{1}}+w_{i_{2}}+\cdots+w_{i_{k}}$, denote by $W-p$ the sequence $\left\{w_{i}: i \neq i_{1}, i_{2}, \ldots, i_{k}\right\}$. Put now $W$ to be the sequence $\left\{z_{i}: i=1,2, \ldots\right\}$.

Let $z \in N, z \neq 0$. Then

$$
\begin{aligned}
\left|\sum\left(z, z_{n}\right)\right| & =\left|\sum\left(z, x_{n}\right)\right|<\infty \quad \text { since } \sum x_{n} \text { converges } \\
\sum\left|\left(z, z_{n}\right)\right| & =\sum\left|\left(z, x_{n}\right)\right|=+\infty \quad \text { since } z \notin M .
\end{aligned}
$$

Hence for any $z \neq 0, z \in N$, and any $T>0$, there exist $p_{1}, p_{2}$ in $P(W)$ such that $\left(z, p_{1}\right) \geqq T$ and $\left(z, p_{2}\right) \leqq-T$. Lemma 4 shows then that $Q(W)$ is dense in $N$. Since $R(W) \supset Q(W)$ (Lemma 3), $R(W)$ is also dense in $N$. It follows then that the set $z+R(W)=\{z+r: r \in R(W)\}$ is also dense for every $z \in N$. The equations (14) and (15) also show that the sets $Q(W-p)$ and $z+R(W-p)$ are dense for every $p \in P(W)$ and $z \in N$. Hence we have shown

(A) for every $w \in N, z \in N, p \in P(W)$ and every $\varepsilon>0$ there exists $r \in z+R(W-p)$ so that

$$
\|w-r\|<\varepsilon .
$$

Choose $w \in N$. We shall now construct a rearrangement of $\sum z_{n}$ which converges to $w$. Let

$$
\varepsilon_{k} \downarrow 0, \quad A_{k}^{2}=\sum_{k}^{\infty}\left\|z_{n}\right\|^{2} \quad \text { and } \quad B_{k}=\sup \left\{\left\|z_{n}\right\|: n \geqq k\right\} .
$$


By the hypothesis of the theorem $A_{k} \downarrow 0, B_{k} \downarrow 0$. We shall define a sequence of elements $w_{k} \in N$ as follows. Let $p_{1}=z_{1}$. Choose $q_{1} \in p_{1}+R\left(z-p_{1}\right)$ such that $q_{1}=z_{1}+u_{1}, u_{1} \in R\left(W-p_{1}\right)$ and so that

$$
\left\|w-p_{1}-u_{1}\right\|<\varepsilon_{1} .
$$

This is possible by (A). We have

$$
u_{1}=\gamma_{1} z_{i_{1}}+\cdots+\gamma_{j} z_{i_{j}}, \quad 0 \leqq \gamma_{i} \leqq 1, \quad 1<i_{1}<\cdots<i_{j} .
$$

Using Lemma 2 we can find $t_{1}=\delta_{1} z_{i_{1}}+\cdots+\delta_{f} z_{i_{j}}, \delta_{i}=0$ or 1 , such that

$$
\left\|u_{1}-t_{1}\right\|^{2} \leqq \sum_{1}^{j}\left\|z_{i_{n}}\right\|^{2} \leqq A_{1}^{2} .
$$

Let $w_{1}=z_{1}+t_{1} \in P(W)$. Clearly from (18), (19) we have

$$
\left\|w-w_{1}\right\| \leqq\left\|w-z_{1}-u_{1}\right\|+\left\|u_{1}-t_{1}\right\| \leqq \varepsilon_{1}+A_{1} .
$$

We now put $p_{2}$ as the first $z$ not used as a summand in $w_{1}$. Choose $q_{2} \in p_{2}+$ $R\left(W-w_{1}-p_{2}\right)$ such that $q_{2}=p_{2}+u_{2}, u_{2} \in R\left(W-\left(p_{1}+w_{1}\right)\right)$ and

$$
\left\|w-w_{1}-p_{2}-u_{2}\right\| \leqq \varepsilon_{2} .
$$

Using Lemma 2 we choose $t_{2} \in P\left(W-\left(p_{2}+w_{1}\right)\right)$ so that

$$
\left\|u_{2}-t_{2}\right\|^{2} \leqq \sum_{2}^{\infty}\left\|z_{i}\right\|^{2}=A_{2}^{2}
$$

Let $w_{2}=p_{2}+t_{2} \in P\left(W-w_{1}\right)$. We get again

$$
\left\|w-w_{1}-w_{2}\right\| \leqq\left\|w-w_{1}-p_{2}-u_{2}\right\|+\left\|u_{2}-t_{2}\right\| \leqq \varepsilon_{2}+A_{2}
$$

Inductively it goes as follows. Suppose $w_{1}, w_{2}, \ldots, w_{n}$ are already defined and satisfy

$$
\begin{gathered}
\left\|w-\left(w_{1}+\cdots+w_{k}\right)\right\| \leqq \varepsilon_{k}+A_{k}, \\
w_{k} \in P\left(W-\left(w_{1}+\cdots+w_{k-1}\right)\right), \quad k=1,2, \ldots, n,
\end{gathered}
$$

(23) $z_{1}, z_{2}, \ldots, z_{k}$ are included as summands in $w_{1}+w_{2}+\cdots+w_{k}, k=1,2, \ldots, n$.

Choose $p_{n+1}$ to be the $z$ with the smallest subscript not included in the sum $w_{1}+\cdots+w_{n}$.

Let $q_{n+1} \in p_{n+1}+R\left(W-\left(p_{n+1}+w_{1}+\cdots+w_{n}\right)\right)$ be such that $q_{n+1}=p_{n+1}+u_{n+1}$ $\in R\left(W-\left(p_{n+1}+\cdots+w_{n}\right)\right)$ and

$$
\left\|w-\left(w_{1}+\cdots+w_{n}+p_{n+1}+u_{n+1}\right)\right\| \leqq \varepsilon_{n+1} .
$$

This is possible by (A). Using Lemma 2 choose $t_{n+1} \in P\left(W-\left(w_{1}+\cdots+w_{n}+p_{n+1}\right)\right)$ so that

$$
\left\|u_{n+1}-t_{n+1}\right\|^{2} \leqq \sum_{n+1}^{\infty}\left\|z_{i}\right\|^{2} \leqq A_{n+1}^{2}
$$


Put $w_{n+1}=p_{n+1}+t_{n+1} \in P\left(W-\left(w_{1}+\cdots+w_{n}\right)\right)$. We have from (24), (25)

$$
\left\|w-\left(w_{1}+\cdots+w_{n+1}\right)\right\| \leqq \varepsilon_{n+1}+A_{n+1} .
$$

Clearly $w_{1}, w_{2}, \ldots, w_{n+1}$ satisfy (21), (22), (23). Thus the sequence $w_{n}$ is defined, every $z_{i}$ is included as a summand and in exactly one $w_{n}$ and

$$
\left\|w-\sum_{1}^{n} w_{i}\right\| \leqq \varepsilon_{n}+A_{n} .
$$

This shows that $\sum w_{n}$ defines a rearrangement of $\sum z_{n}$, say $\sum z_{n}^{\prime \prime}$ such that

$$
w_{n}=z_{i_{n}}^{\prime \prime}+\cdots+z_{i_{n+1}-1}^{\prime \prime}
$$

and

$$
\left\|w-\sum_{1}^{i_{n}+1-1} z_{i}^{\prime \prime}\right\| \leqq \varepsilon_{n}+A_{n} .
$$

Now we rearrange each of the sums $w_{n}$ in such a way that the resulting rearrangement $\sum z_{i}^{\prime}$ will converge to $w$. Let

$$
w_{n}=z_{j_{1}}^{\prime \prime}+\cdots+z_{j_{k}}^{\prime \prime}, \quad n \leqq i_{1}<i_{2}<\cdots<i_{k} .
$$

Using Lemma 5 we can rearrange $z_{j_{1}}^{\prime \prime} \mathrm{s}$ in such a way that

$$
\begin{gathered}
w_{n}=z_{j_{1}}^{\prime}+\cdots+z_{j_{k}}^{\prime} \\
\left\|z_{j_{1}}^{\prime}+\cdots+s_{j_{p}}^{\prime}\right\|^{2} \leqq\left\|z_{j_{1}}^{\prime}\right\|^{2}+\cdots+\left\|z_{k}^{\prime}\right\|^{2}+\left\|w_{n}\right\|\left(\left\|w_{n}\right\|+2 B_{n}\right) \\
\leqq A_{n}^{2}+\left\|w_{n}\right\|\left(\left\|w_{n}\right\|+2 B_{n}\right), \quad p=1,2, \ldots, k .
\end{gathered}
$$

Since

$$
\begin{aligned}
\left\|w_{n}\right\| & \leqq\left\|w-\left(w_{1}+\cdots+w_{n-1}\right)\right\|+\left\|w-\left(w_{1}+\cdots+w_{n}\right)\right\| \\
& \leqq \varepsilon_{n-1}+A_{n-1}+\varepsilon_{n}+A_{n}=d_{n}
\end{aligned}
$$

we have

$$
\left\|z_{j_{1}}^{\prime}+\cdots+z_{j_{p}}^{\prime}\right\|^{2} \leqq A_{n}^{2}+d_{n}\left(d_{n}+B_{n}\right), \quad p=1,2, \ldots, k
$$

Let now $\sum z_{i}^{\prime}$ be the resulting rearrangement. Let $N$ be a positive integer and choose $n=n(N)$ such that

$$
w_{n+1}=z_{i_{1}}^{\prime}+z_{i_{1}+1}^{\prime}+\cdots+z_{i_{k}}^{\prime}, \quad i_{1} \leqq N \leqq i_{k} .
$$

Then

$$
\begin{aligned}
\left\|w-\sum_{1}^{N} z_{i}^{\prime}\right\| & \leqq w-\sum_{1}^{n} w_{i}\|+\| z_{i_{1}}^{\prime}+\cdots+z_{N}^{\prime} \| \\
& \leqq \varepsilon_{n}+A_{n}+\left[A_{n}^{2}+d_{n}\left(d_{n}+B_{n}\right)\right]^{1 / 2} .
\end{aligned}
$$

It is clear that $n(N) \rightarrow \infty$ as $N \rightarrow \infty$; so the right hand side of (33) tends to 0 as $N \rightarrow \infty$. This completes the proof of Theorem 1 . 
3. Examples.

1. $M=\left\{y: \sum\left|\left(x_{n}, y\right)\right|<\infty\right\}$ need not be closed, even if the conditions a-c of Theorem 1 hold.

Let $\left\{e_{n}: n=1,2, \ldots\right\}$ be an orthonormal basis of $L_{2}$. Put

$$
\begin{aligned}
& l_{n}=[(n+2) \log (n+2)]^{-1} \quad x_{n}=(-1)^{n+1} l_{n}\left(e_{1}+e_{2}+\cdots+e_{n}\right) \\
& x=\sum_{i=1}^{\infty} \sum_{j=i}^{\infty}(-1)^{j+1} l_{j} e_{i}=\sum_{i=1}^{\infty} \alpha_{i} e_{i} .
\end{aligned}
$$

It follows that

$$
\left\|x_{n}\right\|^{2}=n l_{n}^{2}<\left[n(\log n)^{2}\right]^{-1}
$$

so $\sum\left\|x_{n}\right\|^{2}<\infty, \sum\left\|x_{n}\right\|=+\infty$. Since $\sum(-1)^{n+1} l_{n}$ is an alternating series, we have the following estimate

$$
\left|\sum_{i=p}^{\infty}(-1)^{n+1} l_{i}-\sum_{i=p}^{p+q}(-1)^{n+1} l_{i}\right|<\left|l_{q+1}\right| .
$$

Hence

$$
\sum_{i=1}^{\infty}\left|\sum_{j=1}^{\infty}(-1)^{n+1} l_{n}\right|^{2}=\sum_{i=1}^{\infty}\left|\sum_{j=1}^{\infty}(-1)^{n+1} l_{n}-\sum_{j=1}^{i=1} l_{n}\right|^{2} \leqq \sum_{i=1}^{\infty}\left|l_{i}\right|^{2}<\infty
$$

so $x \in L_{2}$. Moreover using (36) we obtain

$$
\left\|\sum_{1}^{N} x_{n}-x\right\|^{2}=\left\|\sum_{1}^{N} x_{n}-\sum_{1}^{N} \alpha_{n} e_{n}\right\|^{2}+\left\|\sum_{N+1}^{\infty} \alpha_{n} e_{n}\right\|^{2} .
$$

The last term clearly goes to 0 as $N \rightarrow \infty$. The first term is equal to •

$$
\sum_{n=1}^{N}\left|\sum_{i=n}^{N}(-1)^{n+1} l_{i}-\sum_{i=n}^{\infty}(-1)^{n+1} l_{n}\right|^{2} \leqq \sum_{n=1}^{N}\left|l_{N-1}\right|=N l_{N+1} \rightarrow 0 \quad(N \rightarrow \infty) .
$$

Hence $\sum x_{n}$ converges to $x$ in norm. Put now

$$
\begin{aligned}
t_{i} & =[\log (\log (i+2))]^{-1} \\
y_{1} & =0, \quad y_{k}=t_{1} e_{1}+\sum_{i=1}^{k-1}\left(t_{i+1}-t_{i}\right) e_{i}-t_{k} e_{k}, \quad k \geqq 2, \\
y & =t_{1} e_{1}+\sum_{i=1}^{\infty}\left(t_{i+1}-t_{i}\right) e_{i} .
\end{aligned}
$$

Since

$$
\begin{aligned}
t_{i+1}-t_{i} & =\int_{i}^{i+1} \frac{d}{d x} \frac{1}{\log (\log (x+2))} d x \\
& =-\int_{i}^{i+1}\left[(x+2) \log (x+2)(\log (\log (x+2)))^{2}\right]^{-1} d x
\end{aligned}
$$


so

$$
\left|t_{i+1}-t_{i}\right| \leqq \frac{1}{(i+2) \log (i+2)[\log (\log (i+2))]^{2}}
$$

and hence $y \in L_{2}$. Moreover

$$
\left\|y-y_{k}\right\|^{2} \leqq\left|t_{k}\right|^{2}+\left|t_{k+1}-t_{k}\right|^{2}+\sum_{i=k}^{\infty}\left|t_{i+1}-t_{i}\right|^{2} \rightarrow 0 \quad(k \rightarrow \infty)
$$

so $y_{k}$ converges in norm to $y$. We also note that $\left|\left(x_{n}, y_{k}\right)\right|=0$ for $n>k$, hence $\sum\left|\left(x_{n}, y_{k}\right)\right|<\infty$ for all $k$. However $\left|\left(x_{n}, y\right)\right|=l_{n} t_{n}$ and since

$$
\sum \frac{1}{(i+2) \log (i+2) \log (\log (i+2))}=\infty \quad y \notin M,
$$

this shows that $M$ need not be closed.

2. Next we give an example of a series $\sum x_{n}$ for which $N=L_{2}$, or equivalently $M=\{0\}$. Let $\left\{e_{n}\right\}$ be an orthonormal basis of $L_{2}$. Define

$$
x_{n}^{(k)}=(-1)^{n+1} 2^{-k} n^{-1} e_{k} .
$$

Since $\left\|x_{n}^{(k)}\right\|^{2}=2^{-2 k} n^{-2}$ then $\sum_{n} \sum_{k}\left\|x_{n}^{(k)}\right\|^{2}<\infty$. It is also clear that $\sum_{n, k}\left\|x_{n}^{(k)}\right\|=\infty$. We can order $\left\{x_{n}^{(k)}\right\}$ into a single sequence $\left\{x_{n}\right\}$ such that $\sum x_{n}$ converges. We proceed by induction as follows. Let $x_{1}=x_{1}^{(1)}, x_{2}=x_{1}^{(2)}$ and $x_{3}=x_{2}^{(1)}$. Suppose we have ordered all the elements $x_{n}^{(k)}, n+k \leqq m$, into a sequence $x_{1}, x_{2}, \ldots, x_{N}$ such that if $x_{i}$ corresponds to $x_{n}^{(k)}, x_{j}$ corresponds to $x_{n}^{(k+1)}$ then $i<j$. We put

$$
x_{N+1}=x_{1}^{(m)}, \quad x_{n+2}^{(m)}=x_{2}^{(m-1)}, \ldots, x_{N+j}=x_{j}^{(m-j+1)}, \quad x_{N+m}=x_{m}^{(1)} .
$$

In this way we have ordered all the elements $\left\{x_{n}^{(k)}\right\}$ in such a way that if $x_{i}$ corresponds to $x_{n}^{(k)}$ and $x_{j}$ corresponds to $x_{n}^{(k+1)}$ then $i<j$. Hence for each $k$

$$
\sum_{i}\left(x_{i}, e_{k}\right)=\frac{1}{2^{k}} \sum_{j} \frac{(-1)^{j+1}}{j}=\frac{1}{2^{k}} \log 2=y_{k} .
$$

Put

$$
x=\sum_{k} \frac{1}{2^{k}} \log 2 e_{k}=\sum_{k} y_{k} e_{k} .
$$

We have for any positive integer $N$

$$
\left|\sum_{i=1}^{N}(-1)^{i+1} \frac{1}{2^{k} i}-\frac{1}{2^{k}} \log 2\right| \leqq \frac{1}{2^{k} N} .
$$

Let $N$ be a positive integer and choose $p$ to be the largest integer such that $x_{1}^{(p)}$ is included among $x_{1}, x_{2}, \ldots, x_{N}$. Then by the construction of $x_{i}$ 's

$$
\left\|\sum_{i=1}^{N} x_{i}-x\right\| \leqq\left\|\sum_{1}^{p} x_{i}-\sum_{1}^{p} y_{i} e_{i}\right\|+\left\|\sum_{p+1}^{N} x_{i}-\sum_{p+1}^{N} y_{i} e_{i}\right\|+\left\|\sum_{N+1}^{\infty} y_{i} e_{i}\right\| .
$$


The last sum clearly goes to 0 as $N \rightarrow \infty$. By (44) the first and the second sum are each smaller than

$$
\frac{1}{p} \frac{1}{1}+\frac{1}{p-1} \frac{1}{2}+\frac{1}{p-2} \frac{1}{2^{2}}+\cdots+\frac{1}{1} \frac{1}{2^{p-1}}=S_{p}
$$

which goes to 0 as $p \rightarrow \infty$. Since $p \rightarrow \infty$ as $N \rightarrow \infty$ we have shown that $\sum x_{i}$ converges to $x$ in norm. Let now $y \neq 0, y \in L_{2}, y=\sum b_{k} e_{k}$. Then

$$
\sum\left|\left(x_{i}, y\right)\right| \geqq \sum_{n}\left|\left(x_{n}^{(k)}, y\right)\right|=\sum_{n} \frac{b_{k}}{2^{k} n}=\infty
$$

unless $b_{k}=0$. Hence $M=\{0\}$ and every function in $L_{2}$ can be obtained as a limit of some rearrangement of $\sum x_{i}$.

\section{REFERENCES}

1. M. M. Day, Normed linear spaces, Academic Press, New York, 1962.

2. N. Dunford and J. T. Schwartz, Linear operators, Part I, Wiley, New York, 1958.

3. E. Hille, Analysis. Vol. I, Blaisdell, New York, 1964.

4. E. Steinitz, Bedingt konvergente Reihen und konvexe Systeme, J. Reine Angew. Math. 143 (1913).

State University of New York at Buffalo,

Buffalo, New York 